\title{
Current advances in the application of dynamic NMR studies of DNA/RNA intra-and inter molecular effect on ring inversion rate constants for molecular diagnosis of gum cancer
}

\author{
Alireza Heidari ${ }^{1,2 *}$, Ricardo Gobato ${ }^{3}$ and Lauro Figueroa Valverde ${ }^{4}$ \\ ${ }^{1}$ Faculty of Chemistry, California South University, 14731 Comet St. Irvine, CA 92604, USA \\ ${ }^{2}$ American International Standards Institute, Irvine, CA 3800, USA \\ ${ }^{3}$ Green Land Landscaping and Gardening, Seedling Growth Laboratory, 86130-000, Parana, Brazil \\ ${ }^{4}$ Laboratory of Pharmaco-Chemistry, Faculty of Chemical Biological Sciences, University Autonomous of Campeche, Humberto Lanz Cárdenas S/N, Colonia Ex \\ Hacienda Kalá, P. O. Box 24085, Campeche, México
}

\begin{abstract}
All oncologists are marked by fundamental biochemical changes at the molecular, cellular, and tissue level. The identification and further understanding of these changes would allow improved prevention, prognosis, diagnosis and treatments, as well as overall management and disease survival. The potential of dynamic NMR biospectroscopy in molecular diagnostics relies on its ability to determine and characterize the unique fingerprint of a sample at the biochemical level for dynamic NMR studies of DNA/RNA intra-and inter molecular effect on ring inversion rate constants for molecular diagnosis of gum cancer.
\end{abstract}

\section{Introduction}

The high incidence rate of gum cancer as well as its progressiveness has led researchers to continually examine and pursue better prevention, diagnosis, prognosis, and treatment techniques to decrease mortality rates and comorbidity from the disease. As highlighted, not only does dynamic NMR studies of DNA/RNA intra-and inter molecular effect on ring inversion rate constants for molecular diagnosis of gum cancer have the potential to identify cancerous and precancerous tissue, it also has the ability to probe deeper into the disease fingerprint to elucidate its underlying mechanisms. By implementing variations of the technique to study a wide range of samples such as commercially available cell lines, FFPP sections, and in vivo and ex vivo tissue, the potential of dynamic NMR studies of DNA/RNA intra-and inter molecular effect on ring inversion rate constants for molecular diagnosis of gum cancer as a viable option for a future diagnostic technology of gum cancer and other disease states has been shown. In gum cancer, a number of different factors including HPV infection, hormonal imbalances, and inflammatory infection have already been reported to influence dynamic NMR studies of DNA/RNA intra-and inter molecular effect on ring inversion rate constants for molecular diagnosis of gum cancer. Whilst these could be seen as limitations, they actually prove the sensitivity of the technique and support additional evidence generated by other approaches such as proteomics and oncology [1-10].

\section{Results and discussion}

A far greater body of evidence is still required before this technology can make head way in a clinical setting. For instance, the engagement of the clinical community in supporting more comprehensive studies both in vivo and ex vivo, cognizant of all variables and considering a wide range of controls, gathered from a representative spectrum of the population would be vital to take the technique a step closer to gum cancer diagnosis. If such studies could be undertaken and the reliability of the technique proven, dynamic NMR studies of DNA/RNA intraand inter molecular effect on ring inversion rate constants for molecular diagnosis of gum cancer could have a real future in clinical diagnostics of gum cancer and similar ontologies (Figure 1).

\section{Conclusion}

However, lack of information regarding which data was considered for the sensitivity and specificity values reported, as well as the lack of positive and negative predictive values, calls for the standardization in the reporting of these important performance measurements. Likewise, sample handling and processing ought to be reported as it can influence dynamic NMR studies of DNA/RNA intra-and inter molecular effect on ring inversion rate constants for molecular diagnosis of gum cancer profiles. Finally, whilst acknowledging the exploratory

*Correspondence to: Alireza Heidari, Faculty of Chemistry, California South University, 14731 Comet St. Irvine, CA 92604; American International Standards Institute, Irvine, CA 3800, USA, E-mail: Scholar.Researcher.Scientist@gmail. com; Alireza.Heidari@calsu.us; Central@aisi-usa.org

Key words: dynamic NMR studies, DNA/RNA, intra-and inter molecular effect, ring inversion, rate constants, molecular diagnosis, gum cancer

Received: December 31, 2020; Accepted: January 26, 2021; Published: January 29,2021 
Heidari A (2021) Current advances in the application of dynamic NMR studies of DNA/RNA intra-and inter molecular effect on ring inversion rate constants for molecular diagnosis of gum cancer

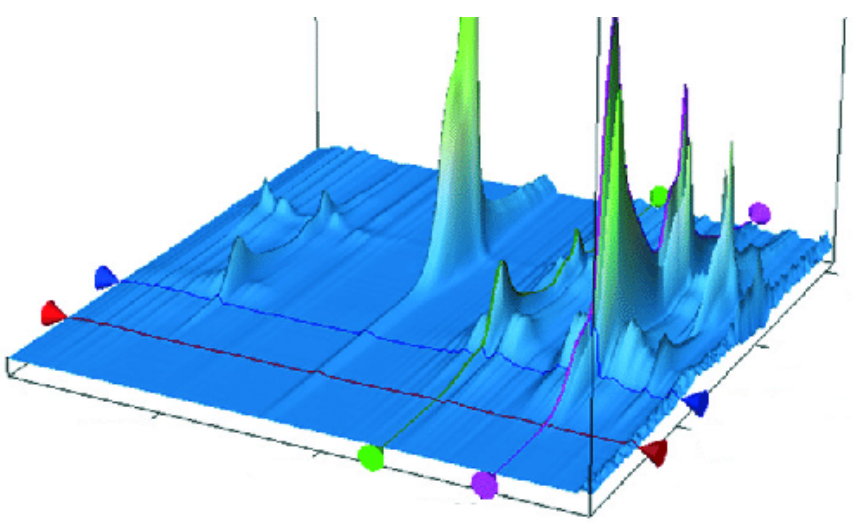

Figure 1. Simulation of DNA/RNA intra-and inter molecular effect on ring inversion rate constants for molecular diagnosis of gum cancer

nature of most studies and the difficulty in obtaining patient samples, a frank criticism is the small sample size of most reported studies. Although biospectroscopically significant due to the high number of biospectroscopic measurements, more samples are required to assess the biological and pathological relevance and reproducibility for dynamic NMR studies of DNA/RNA intra-and inter molecular effect on ring inversion rate constants for molecular diagnosis of gum cancer.

\section{Acknowledgement}

This study was supported by the Cancer Research Institute (CRI) Project of Scientific Instrument and Equipment Development, the National Natural Science Foundation of the United Sates, the International Joint BioSpectroscopy Core Research Laboratory Program supported by the California South University (CSU), and the Key project supported by the American International Standards Institute (AISI), Irvine, California, USA.

\section{References}

1. Heidari, C. Brown, "Study of Composition and Morphology of Cadmium Oxide (CdO) Nanoparticles for Eliminating Cancer Cells", J Nanomed Res., Volume 2, Issue 5, 20 Pages, 2015.

2. A. Heidari, C. Brown, "Study of Surface Morphological, Phytochemical and Structural Characteristics of Rhodium (III) Oxide $\left(\mathrm{Rh}_{2} \mathrm{O}_{3}\right)$ Nanoparticles", International Journal of Pharmacology, Phytochemistry and Ethnomedicine, Volume 1, Issue 1, Pages 15-19, 2015.

3. A. Heidari, "An Experimental Biospectroscopic Study on Seminal Plasma in Determination of Semen Quality for Evaluation of Male Infertility", Int J Adv Technol 7: e007, 2016.

4. A. Heidari, "Extraction and Preconcentration of N-Tolyl-Sulfonyl-PhosphoramidSaeure-Dichlorid as an Anti-Cancer Drug from Plants: A Pharmacognosy Study", J Pharmacogn Nat Prod 2: e103, 2016.

5. A. Heidari, "A Thermodynamic Study on Hydration and Dehydration of DNA and RNA-Amphiphile Complexes", J Bioeng Biomed Sci S: 006, 2016.

6. A. Heidari, "Computational Studies on Molecular Structures and Carbonyl and Ketene Groups' Effects of Singlet and Triplet Energies of Azidoketene $O=C=C H-N N N$ and Isocyanatoketene $O=C=C H-N=C=O$ ", $\mathrm{J}$ Appl Computat Math 5: e142, 2016.

7. A. Heidari, "Study of Irradiations to Enhance the Induces the Dissociation of Hydrogen Bonds between Peptide Chains and Transition from Helix Structure to Random Coil Structure Using ATR-FTIR, Raman and 'HNMR Spectroscopies", J Biomol Res Ther 5: e146, 2016.

8. A. Heidari, "Future Prospects of Point Fluorescence Spectroscopy, Fluorescence Imaging and Fluorescence Endoscopy in Photodynamic Therapy (PDT) for Cancer Cells", J Bioanal Biomed 8: e135, 2016.

9. A. Heidari, “A Bio-Spectroscopic Study of DNA Density and Color Role as Determining Factor for Absorbed Irradiation in Cancer Cells", Adv Cancer Prev 1: e102, 2016.

10. A. Heidari, "Manufacturing Process of Solar Cells Using Cadmium Oxide (CdO) and Rhodium (III) Oxide $\left(\mathrm{Rh}_{2} \mathrm{O}_{3}\right)$ Nanoparticles", J Biotechnol Biomater 6: e125, 2016.

Copyright: (C2021 Heidari A. This is an open-access article distributed under the terms of the Creative Commons Attribution License, which permits unrestricted use, distribution, and reproduction in any medium, provided the original author and source are credited. 